\title{
P138 Modelling and Simulation of Pressure Re-reflections at the Aortic Valve Using Difference Equations
}

\author{
Bernhard Hametner ${ }^{1}$, Hannah Kastinger ${ }^{2}$, Siegfried Wassertheurer ${ }^{1}$ \\ ${ }^{1}$ Center for Health and Bioresources, AIT Austrian Institute of Technology, Vienna, Austria \\ ${ }^{2}$ Institute for Analysis and Scientific Computing, Vienna University of Technology, Vienna, Austria
}

\section{ABSTRACT}

Introduction: The concept of re-reflections of blood pressure waves at the heart has been neglected in most models and is only poorly understood so far. Therefore, the aim of this work was to develop a simple mathematical model which is capable to represent the effects of wave travel, wave reflection as well as re-reflection at the aortic valve.

Methods: A mathematical model based on difference equations has been developed. The equation for the backward travelling pressure waves includes several terms, where the forward pressure is multiplied by a specific reflection coefficient and is delayed by a specific period of time corresponding to the location of the reflection site and the stiffness of the travel path. The equation for the forward travelling waves includes an input pressure coming from the heart as well as the backward pressure multiplied by a time-dependent re-reflection coefficient.

Results: After identifying suitable distal reflection parameters to get a realistic pressure wave, sensitivity analyses on the timedependent re-reflection coefficient were performed. Exemplarily, the re-reflection coefficient was held at the level of 1 during diastole (aortic valve closed), while it was varied from 0.6 to 0.9 during systole, see Figure 1 .

Conclusion: With a simple difference equation model, aortic pressure waves can be simulated adequately. The choice of the re-reflection coefficient at the aortic valve strongly influences the results, both regarding pressure level and wave shape. This indicates that re-reflections should be incorporated into models of wave transmission as well as in methods of arterial pulse wave analysis.

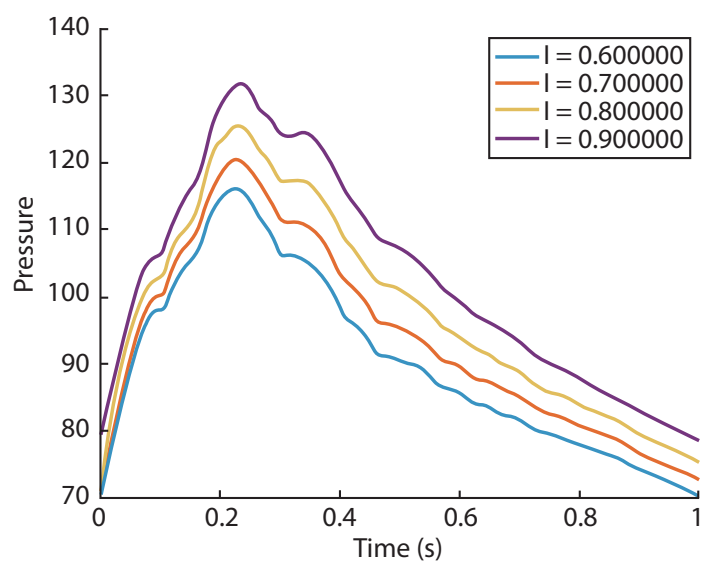

Figure 1

(c) 2019 Association for Research into Arterial Structure and Physiology. Publishing services by Atlantis Press International B.V. This is an open access article distributed under the CC BY-NC 4.0 license (http://creativecommons.org/licenses/by-nc/4.0/). 